\title{
Sindrome de Burnout en personal de salud durante la pandemia COVID-19: un semáforo naranja en la salud mental
}

\author{
Burnout syndrome in healthcare personnel during the COVID-19 \\ pandemic: An orange traffic light in mental health
}

\begin{abstract}
Arturo Juárez García ${ }^{1}$
Forma de citar: Juárez-García A. Síndrome de Burnout en personal de salud durante la pandemia COVID-19: un semáforo naranja en la salud mental. Salud UIS. 2020; 53(4): 432-439. doi: https://doi.org/10.18273/revsal.v52n4-2020010 @ (1)

\section{Resumen}

La pandemia de COVID-19 es un reto enorme para la salud pública y la economía mundial; también es un reto mayor para la salud mental, siendo uno de los grupos potencialmente más afectados el personal de salud. Uno de los efectos que ya se observa entre los trabajadores de este grupo ocupacional es el síndrome de burnout. En este artículo se revisa el origen del síndrome de burnout entre el personal sanitario, su importancia en el contexto de la pandemia y se proponen estrategias para disminuir o controlar su ocurrencia.
\end{abstract}

Palabras clave: COVID-19; Salud mental; Burnout; Personal sanitario.

\begin{abstract}
The COVID-19 pandemic is a huge challenge for public health and the global economy; It is also a major challenge for mental health, with health personnel being one of the potentially most affected groups. One of the effects that is already observed among workers in this occupational group is burnout syndrome. This article reviews the origin of burnout syndrome among health personnel, its importance in the context of the pandemic, and strategies are proposed to reduce or control its occurrence.
\end{abstract}

Key words: COVID-19; Mental health; Burnout; Healthcare personnel.

\footnotetext{
1. Universidad Autónoma del Estado de Morelos, México.

Correspondencia: Dirección: Pico de Orizaba No. 1 esq. Popocatépetl, Col. Volcanes, Cuernavaca, Morelos, México. Correo electrónico: arturojuarezg@hotmail.com
} 


\section{Introducción}

Es innegable que el brote por el coronavirus SARSCOV2 ha significado un parteaguas en la historia de la humanidad, pues sus efectos han sido devastadores en muy poco poco tiempo. El 11 de marzo del 2020 la Organización Mundial de la Salud (OMS) declaró a la enfermedad COVID-19 (ocasionada por el virus SARSCOV2) como una pandemia, y tan solo dos semanas después ya se había propagado a 114 países, muchos de ellos adoptando cuarentenas totales con impactos económicos incalculables. En los primeros días de Julio en que se elabora este escrito (poco más de seis meses después de la primera notificación en Wuhan China) se contabilizan más de 11 millones de personas contagiadas en el mundo y más 540000 muertos por esta enfermedad, en medio de una economía mundial severamente dañada ${ }^{1}$.

En este contexto, la mayor preocupación de las naciones durante la pandemia ha sido el control de la carga hospitalaria y la demanda de servicios de salud. En América Latina en particular, esta epidemia puso al descubierto déficits de los sistemas sanitarios que ya eran preexistentes, los que revelaban desde antes una capacidad de respuesta limitada ante la demanda poblacional, a pesar de muchas reformas recientes ${ }^{2,3}$. De esta forma, además del riesgo implícito por exposición al coronavirus, la gran carga de trabajo y las demandas psicoemocionales a las que han sido expuestos los trabajadores de salud durante esta pandemia, son indescriptibles e inconmensurables y representan uno de los retos más evidentes en el tema de los factores de riesgo psicosocial en el sector salud en Latinoamérica y el mundo.

Aún se sigue estudiando el impacto global que la pandemia COVID 19 ha tenido en el estrés y la salud mental, pero la Organización de las Naciones Unidas ha advertido que provocará un aumento en el número y severidad se los problemas de salud mental en cientos de millones de personas y que dichos problemas tienen riesgo de ser ignorados o subestimados ${ }^{4}$. La OMS por su parte, ha confirmado que el impacto de la pandemia en la salud mental es extremadamente preocupante y que los trabajadores de cuidado de la salud que están en la primera línea de atención al COVID-19, son particularmente vulnerables por las altas cargas de trabajo, el riesgo de infección y las decisiones de vida o muerte que tienen que tomar, entre otras cosas 5 .
Hasta ahora, poco se han estudiado las interrelaciones y mecanismos antecedentes de distintos indicadores de salud mental, donde el síndrome de burnout juega un rol relevante. Por ello, el objetivo de este escrito es describir brevemente las demandas psicosociales y los efectos en la salud mental en profesionales de la salud que se están documentando durante la pandemia de COVID-19, y mostrar el rol del síndrome de burnout en estos procesos y su importancia como indicador para la prevención secundaria.

\section{Exigencias psicosociales y salud mental en personal de salud durante la pandemia}

Desde antes de la pandemia por la enfermedad COVID-19, era bien sabido que los profesionales de la salud están comúnmente expuestos a diversos estresores psicosociales por sus tareas cotidianas, entre los que se encuentran: las altas cargas de trabajo, el bajo control, bajo apoyo social, demandas emocionales y físicas, desequilibro entre esfuerzo y recompensa, liderazgo negativo, violencia laboral, falta de equipos y materiales, horarios y jornadas prolongadas, entre otros $^{6-11}$.

Con la llegada de la pandemia, queda claro que todos estos factores se han incrementado de una manera $u$ otra, e incluso se han sumado otros estresores que son propios de situaciones de epidemias. Al respecto, la Organización Internacional del Trabajo (OIT) $)^{12}$ señala que los principales factores psicosociales de estrés durante una pandemia para personal que se encuentra en la primera línea de emergencia (trabajadores sanitarios, personal de laboratorio, trabajadores funerarios, operadores de ambulancia, empleados de limpieza y manejo de desechos, policías, militares, bomberos, entre otros), son: el temor por el bienestar propio o de familia y compañeros, falta de equipo de protección personal, aislamiento, falta de apoyo social o de tejido social, tensión entre los protocolos de seguridad establecidos y el deseo de cuidar o ayudar a personas, dificultades para mantener estilos de vida saludables, aumento de exposición a la estigma social, discriminación e incluso violencia por otras personas. La misma OIT señala que las reacciones comunes a estos factores se traducen en estrés negativo, estado de ánimo bajo, baja motivación, mayor fatiga y repercusiones en la depresión, ansiedad y otros efectos graves en la salud mental. 
Vale la pena señalar, que algo que se puede agregar como característico de esta pandemia en particular, es la incertidumbre que envuelve el desconocimiento que aún existe de los mecanismos de contagio, afectación y resistencias del virus SARS-COV2, así como la falta de certeza sobre la duración que tendrá la crisis, la llegada de terapias y vacunas eficaces, la disponibilidad de recursos y equipos, la ética en la toma de decisiones sin información, e incluso situaciones relacionadas a la preocupación por el bienestar de la familia que los han llevado a un distanciamiento social atípico con los seres queridos ${ }^{13,14}$.

Dado lo anterior, las consecuencias en la salud mental por estos estresores psicosociales durante la pandemia de COVID-19 no se han hecho esperar. El estudio ya clásico de Lai, et al. ${ }^{15}$ en 1257 trabajadores de 34 hospitales en China, encontró que 50,4\% de los participantes presentaba síntomas de depresión, $44,6 \%$ de ansiedad, $35 \%$ de insomnio y $71,5 \%$ de distrés. En Francia se reportaron incrementos en las adicciones e intentos de suicidio ${ }^{16}$. Otro estudio chino encontró, que $35,6 \%$ de los trabajadores de la salud presentaban ansiedad generalizada y el $23,6 \%$ problemas de sueño ${ }^{17}$. En italia ${ }^{18}$ se encontraron prevalencias similares en estos trabajadores, aunque adicionalmente ubicó 49,38\% de síntomas altos de estrés postraumático.

En contraparte a estos hallazgos, otro estudio en Singapur ${ }^{19}$ presentó prevalencias más bajas: 14,5\% para ansiedad, 8,9\% para depresión, 6,6\% para distrés y $7,7 \%$ para estrés postraumático. La explicación de los autores sobre estos niveles más bajos, la atribuyen a la experiencia previa de Singapur en el manejo de salud mental en personal de salud, ganada a partir de la epidemia del síndrome severo respiratorio agudo (SARS). Sin embargo, una variable que no debe desestimarse, es la resistencia del personal de salud a expresar su vulnerabilidad ante problemas de salud mental o a recibir apoyo psicológico, lo que ha ocurrido en otros tamizajes durante esta pandemia ${ }^{15,20}$.

En México, algunas encuestas institucionales encontraron que $7 \%$ de los trabajadores de la salud han recibido agresiones en la vía pública ${ }^{21}, 40 \%$ reconoce que se ha ausentado por estrés y el $35 \%$ ha considerado renunciar durante esta contingencia ${ }^{22}$. Asimismo, hallazgos preliminares de un estudio en personal de salud mexicano, que labora en hospitales de distintas regiones durante la pandemia de COVID-1923, ha encontrado que a $47 \%$ le gustaría recibir apoyo psicológico, $27,4 \%$ presenta síntomas de ansiedad, 16,9\% de depresión y 44,1\% de estrés, pero la frecuencia más alta se encuentra en el efecto psicológico del desgaste emocional o burnout con un 47,6\%. Esto es relevante, pues existen pocos estudios que aborden el síndrome de burnout durante esta pandemia a pesar de su rol en el contexto de las afectaciones de la salud mental y su prevención, motivo del siguiente apartado.

\section{El burnout y su importancia en el contexto de la pandemia}

Aunque los desórdenes de ansiedad, depresión o estrés postraumático han sido los más estudiados hasta ahora durante la pandemia COVID-19, es evidente que no capturan totalmente todas las dimensiones y mecanismos de la salud mental en los trabajadores de la salud ante la actual contingencia sanitaria mundial, de forma que, entre más sean las dimensiones exploradas y mayor sea la comprensión de sus interconexiones entre sí y sus posibles antecedentes, se tendrían mejores posibilidades de diseñar intervenciones más efectivas.

En particular, el síndrome de burnout o desgaste emocional es un efecto psicológico negativo generado por condiciones de trabajo adversas y recientemente la OMS lo definió como: "un síndrome que resulta de estresores crónicos del trabajo que no han sido afrontados exitosamente. Se encuentra caracterizado por tres dimensiones: 1) sentimientos de deterioro de energía o agotamiento, 2) distanciamiento mental del trabajo o sentimientos negativos o indiferencia relacionada al trabajo; y 3) eficacia profesional reducida. El burnout se refiere especificamente a un fenómeno del contexto ocupacional y no debe ser aplicado para describir experiencias en otras áreas de la vida"24. Aunque esta concepción coincide con la definición más popular expresada por Maslach ${ }^{25}$, cada vez se reconoce más, que un patrón bidimensional solo con los síntomas de agotamiento e indiferencia (al trabajo o a las personas), pueden ser suficientes para considerarse que estamos frente a un caso de burnout ${ }^{26,27}$. Teóricamente, esto coincide con el modelo circunflejo de afecto o bienestar psicológico de Russell ${ }^{28,29}$, donde se representa el conjunto de relaciones mutuas de sentimientos o afectos en un espacio de orden circular formado por dos dimensiones bipolares: alta-baja activación (eje de energía) y agradabilidad-desagradabilidad (eje del placer), lo que posibilita cuatro efectos psicológicos posibles. El burnout se ubica en el cuadrante de la combinación de baja activación o energía, y bajo placer o agradabilidad, lo que puede ser de utilidad práctica para un rápido tamizaje (Figura 1). 


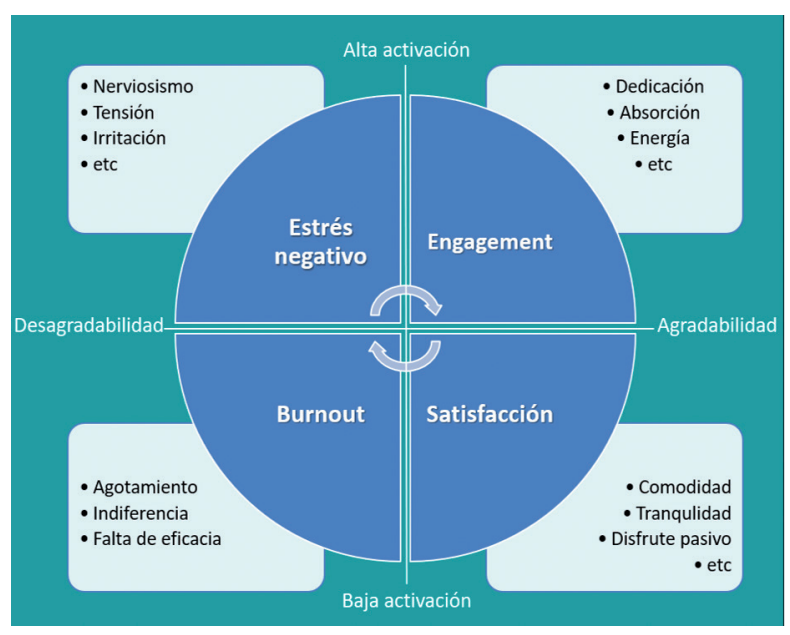

Figura 1. Modelo Circunflejo de Bienestar Psicológico. (adaptado de Russell, 1980) ${ }^{28}$.

En el 2019 la OMS incluyó al síndrome de burnout en la lista de la Clasificación Internacional de Enfermedades (CIE-11) (QD85 Burn-out), sin embargo, no lo incluye como una condición médica, sino como un fenómeno ocupacional dentro de los "problemas asociados con el empleo o el desempleo" y se describe también en el capítulo "factores que influyen en el estado de salud o que necesitan atención de servicios médicos", pero no propiamente clasificado como enfermedad o trastorno de salud ${ }^{30}$.

Esto converge plenamente con las visiones psicosociales originales del fenómeno, donde el burnout se vislumbra más como una alteración del bienestar psicosocial, que como una enfermedad médica dentro del marco rígido y hegemónico del modelo biomédico ${ }^{31,32}$. Esta acepción del burnout es importante, no solo por la necesidad de una concepción más integral, real y justa de sus características, sino por sus implicaciones en los mecanismos de afectación, así como de las acciones de prevención que buscan evitar su ocurrencia y sus complicaciones por las consecuencias en la cadena causal del proceso salud-enfermedad.

Aunque el burnout no es una enfermedad como tal, es bien sabido que sus consecuencias o efectos sí comprenden trastornos de la salud mental y el bienestar subjetivo, pero también deterioros objetivos del sistema musculoesquelético, respiratorio, cardiovascular, endócrino e inmunológico ${ }^{33,34}$, así como otros efectos en el desempeño laboral de personal de salud, que se extienden al ausentismo, errores médicos y más días de recuperación de pacientes, entre muchas otras disfunciones adicionales ${ }^{35}$.
Existe una aceptación generalizada, de que por un lado, el burnout es un mediador patógeno entre el estrés por exposición a diversos factores psicosociales laborales, y por el otro, los múltiples trastornos mentales y físicos que se presentan como consecuencia de éste, y que su proceso forma parte de los mecanismos del estrés negativo. Desde la teoría madre del estrés, Selye ${ }^{37}$ habló de tres fases de lo que llamó el síndrome general de adaptación: alarma, resistencia y agotamiento, siendo el último, un elemento compatible a la visión psicológica del burnout. De modo que primero es el estrés y luego el burnout, y lo que ocurre después en ese proceso, entra en el terreno propio de la enfermedad y el trastorno, que por sus características, es menos reversible que el estrés negativo o el burnout (aunque el estrés negativo es también de más pronta recuperación que el burnout).

Con lo anterior, es posible alinear el proceso del burnout a los paradigmas de prevención primaria, secundaria y terciaria en salud pública ${ }^{38}$ y aplicarlo al contexto actual de la pandemia por COVID-19. De forma que, las acciones de prevención primaria corresponden a una atención a los factores causales o predisponentes, en este caso, las condiciones estresantes del trabajo que están enfrentando los profesionales de la salud y han sido antes mencionados; la prevención secundaria se enfocaría a la sintomatología del estrés y burnout como proceso previo a trastornos más severos y estables, por lo que su monitoreo actual se vuelve trascendente; y finalmente, la prevención terciaria implicaría medidas orientadas al tratamiento ad integrum de trastornos o enfermedades clínicamente más severas y de menor reversibilidad, mediante distintas estrategias orientadas a la rehabilitación. Así, es posible un modelo de semaforización donde los procesos de estrés y burnout son claves en la prevención más temprana de trastornos severos de salud mental y física por causa de la pandemia de COVID-19, como se muestra en la Figura 2.

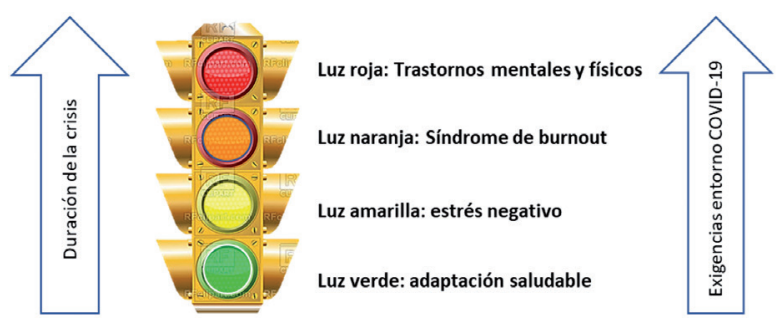

Figura 2. Semaforización del proceso psicosocial ante el COVID-19: el burnout una luz naranja.

Actualmente se estima que la pandemia por COVID-19 tiene cuatro olas o huellas en la salud de la humanidad ${ }^{39}$; la primera, es el impacto directo de morbi-mortalidad 
por contagios, la segunda es el impacto por la limitación los recursos o atenciones a condiciones de salud que no son COVID-19, la tercera se refiere a impactos que implican la interrupción de atención a pacientes con diagnóstico de enfermedades crónicas, y la cuarta ola que se advierte como la más grande, refiere al incremento sustancial de casos de burnout, trastornos mentales y traumas psíquicos derivados de todos los sucesos sociales y económicos relacionados con la pandemia. De este modo, los profesionales de la salud mental son los próximos a estar en la primera línea de atención, por lo que deben diseñarse estrategias tempranas y programas preventivos exhaustivos para este daño colateral producido por esta crisis sanitaria, el cual apenas comienza y amenaza con una fase aguda e intensa.

Esta cuarta ola, equivalente a una pandemia de salud mental no está exenta para los trabajadores de la salud, y la depresión y ansiedad severas así como el estrés postraumático serán muy comunes, por lo que el monitoreo y atención temprana del estrés negativo y el burnout, son estrategias ineludibles desde la prevención secundaria que deben aminorar los impactos en la salud mental causados por el COVID-19 en todo el planeta.

\section{¿Cuáles son las estrategias idóneas para disminuir o controlar el burnout en medio de la pandemia por COVID-19?}

En principio, es importante señalar que no existe mejor inicio de intervención que un buen diagnóstico, y en esta medida, el monitoreo y evaluación del síndrome de burnout en los profesionales de la salud, así como sus posibles factores asociados, son cruciales durante esta crisis de acuerdo con las lógicas de prevención antes descritas. Entre los pocos estudios publicados sobre el síndrome de burnout en trabajadores de la salud en tiempos de COVID-19 se ubican como posibles factores predisponentes: a las privaciones de sueño motivadas por las exigencias actuales ${ }^{40}$, el riesgo biológico ocupacional intrínseco, la respuesta tardía de autoridades, procesos ineficientes e impactos económicos que se viven ${ }^{41}$, la cuarentena obligada a la que tienen que someterse los trabajadores de la salud fuera de casa y los dilemas éticos en la toma de decisiones de atención a pacientes ${ }^{42}$. Sin embargo, un estudio ha mostrado que los estresores vinculados al burnout con más "rating" son la falta de equipo de protección personal, el miedo al contagio de COVID-19 y el miedo de contagiar a los familiares ${ }^{43}$. La exploración preliminar hecha en México ha encontrado una asociación del burnout del personal de salud con el padecimiento de una enfermedad crónica, tener síntomas o haber sido diagnosticados de COVID-19, haber perdido un colega o familiar por COVID-19, percibir mayor temor y daños por riesgo de contagio, y el número de semanas y pacientes atendidos por COVID-1923.

En la lógica de prevención primara, los estresores antes descritos resultan ser los retos primarios de atención, por lo que las medidas de intervención iniciales debieran dirigirse a combatir estos estresores u otorgar recursos del entorno para disminuir su impacto, lo que requiere de un involucramiento institucional que va más allá del esfuerzo individual o interno de los trabajadores de la salud por "controlar su estrés". En el marco de la disciplina de psicología de la salud ocupacional y desde las perspectivas teórico-metodológicas del burnout, se ha señalado señalado que existe un sobre-énfasis en la relevancia de las intervenciones psicológicas individuales, las cuales pueden ser insuficientes o tener efectos solo a corto plazo y deben ser complementadas con intervenciones organizacionales o contextuales para asegurar un mayor y más sostenible beneficio ${ }^{44,45}$. En este sentido, estrategias institucionales que implican cambios y mejoras en procesos organizacionales y que otorgan recursos y apoyos a los trabajadores de la salud para enfrentar el estrés negativo, también deben ser implementadas en conjunto con los programas de asistencia psicológica grupal o individual que actualmente se están implementando para atender la salud mental de los trabajadores de la salud.

Adicionalmente, si existe un contexto macro-social en el que se desarrollan normativas nacionales o políticas sectoriales dirigidas a la salud mental, la eficacia de las estrategias de intervención adoptadas cobran mayor fuerza. Pudiera pensarse que iniciativas de esta índole implican cambios de largo plazo, sin embargo, ninguno como los cambios sociales abruptos que ha provocado el COVID-19 serían comparables a los que se necesitan para prevenir los posibles problemas venideros de la salud mental. Una opción adicional en estos casos es simplemente hacer uso de leyes y normas sobre factores de riesgo psicosocial laboral que ya existen en algunos países, como es el caso de Colombia y México. De este modo, una jerarquía de distintos niveles de prevención o intervención que pueden operar conjuntamente y generar mayor impacto se muestran en la Figura 3. 


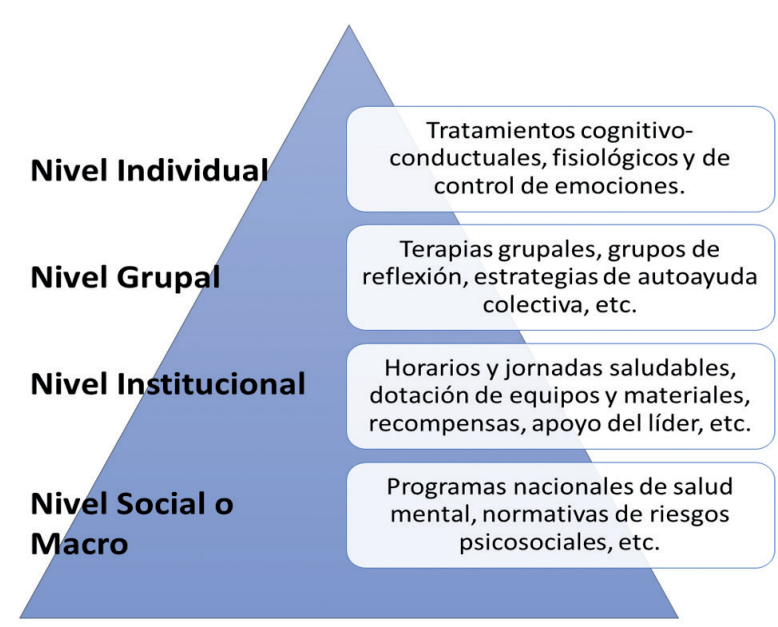

Figura 3. Niveles de intervención en burnout y salud mental en trabajadores de la salud ante COVID-19.

Algunas intervenciones sobre salud mental en trabajadores de la salud ante la pandemia por COVID-19 disponibles en la literatura reciente, sugieren cierta efectividad, por ejemplo, Lai, et al..$^{15}$ implementaron un programa de atención con cuatro equipos: equipo de respuesta psicológica (diseña intervenciones), equipo de soporte técnico (asegura materiales), equipo de asistencia psicológica directa y equipo de asistencia vía telefónica. Sasangohar, et al. ${ }^{41}$ sugiere información continua on line al personal sobre disponibilidad de equipo de protección personal y procesos diversos durante la contingencia aprovechando la tecnología, así como seguimiento emocional a los trabajadores de la salud. Desde las intervenciones individuales, Fessell y Cherniss $^{46}$ implementaron actividades breves de mindfulness, ejercicios breves de relajación y diarios de agradecimientos a las que llamaron "microprácticas" con buen grado de éxito. Lozano ${ }^{47}$ sugiere intervenciones de corte más organizacional: asegurar el descanso de los trabajadores de la salud mediante la rotación de puestos, organización de turnos, días libres, espacio para dormir y alimentarse, entre otros. Finalmente, Ornell, et al. ${ }^{48}$, señalan que es imperativo la amplia inversión en investigación prevención y tratamiento de salud mental en trabajadores sanitarios por parte del gobierno y agencias sanitarias, quienes tienen una importante responsabilidad en ello, lo que implicaría iniciativas a nivel macro social que asegurarían mayores impactos positivos.

\section{Discusión y conclusiones}

Son muchas las lecciones que esta pandemia nos ha dado, entre ellas que muchos sectores productivos se valoran mediante el impacto económico que tienen en el producto interno bruto en cada nación, pero con la pandemia por COVID-19 queda claro que el sector salud tiene una contribución incalculable y es simplemente indispensable y estratégica en la sociedad. Otra lección es que las enfermedades infecto-contagiosas, lejos de quedar en el pasado con los avances de la medicina como se creía hace unas décadas, han llegado para quedarse y el sector salud debe estar preparado permanentemente para epidemias como la experimentada con el COVID-19.

El personal sanitario es la parte más débil en la cadena de atención a la pandemia, no solo porque las estadísticas de contagio apuntan que hasta $20 \%$ de lo pacientes infectados por COVID-19 son personal de salud ${ }^{49}$, sino porque estos trabajadores al estar en primera línea, están enfrentado una variedad amplia de demandas psicosociales muy altas que los posiciona en una alta vulnerabilidad al estrés y alteraciones mentales en general, deteriorando su calidad de vida, su entorno y su capacidad funcional. Esto conforma claramente un círculo vicioso entre riesgos biológicos y psicosociales, con impacto a su persona, su trabajo y la sociedad en general.

El síndrome de burnout forma parte del entramado del estrés crónico laboral, y como posible respuesta inicial de alguna alteración a la salud mental, su monitoreo y control expedito se enmarca en las estrategias de prevención (secundarias), lo que lo convierte en un indicador (semáforo naranja) de evaluación idóneo y útil de salud mental en los centros hospitalarios, antes de adoptar estrategias más centradas en la rehabilitación. La cuarta ola de la pandemia por COVID-19 se refiere a una epidemia de salud mental que aún no comienza y el enfoque preventivo es más importante que nunca.

Una estrategia integral de prevención de burnout y alteraciones mentales en general debe adoptar medidas a cuatro niveles: aquellas basadas en la creación de políticas, guías y programas gubernamentales nacionales, medidas de corte organizacional $o$ institucional, medidas grupales y medidas individuales. Todas estas medidas deben estar alineadas y deben incluir el monitoreo continuo, aprovechando la tecnología, y con intervenciones específicas basadas en la evidencia.

\section{Referencias}

1. World Health Organization. WHO Coronavirus Disease (COVID-19) Dashboard | WHO Coronavirus Disease (COVID-19) Dashboard, https://covid19.who.int/ 
2. Figueras J, Musgrove P, Carrin G, Durán A. Retos para los sistemas sanitarios de Latinoamérica: Qué puede aprenderse de la experiencia europea? Gac Sanit. 2002; doi: 10.1016/S0213-9111(02)71628-2

3. Vargas I, Vázquez ML, Jané E. Equidad y reformas de los sistemas de salud en Latinoamérica. Cad Saude Publica. Epub ahead of print 2002. doi: 10.1590/s0102-311x2002000400003

4. UN (United Nations). Policy Brief : COVID-19 and the Need for Action on Mental Health EXECUTIVE SUMMARY. 2020; https://www.un.org/sites/un2. un.org/files/un_policy_brief-covid_and_mental_ health_final.pdf

5. World health Organization. Substantial investment needed to avert mental health crisis. Who.Int 2020; 5-7.

6. Birhanu M, Gebrekidan B, Tesefa G, Tareke M. Workload determines workplace stress among health professionals working in felege-hiwot referral Hospital, Bahir Dar, Northwest Ethiopia. J Environ Public Health. 2018; doi: 10.1155/2018/6286010

7. Bourbonnais R, Brisson C, Vézina M. Long-term effects of an intervention on psychosocial work factors among healthcare professionals in a hospital setting. Occup Environ Med. 2011; 68(7): 479-486. doi: 10.1136/oem.2010.055202

8. Lavoie-Tremblay M, Sounan C, Lavigne GL, Bonin JP, Lesage AD, Denis P, et al. The psychosocial work environment and evidence utilization by health professionals. Can J Nurs Res. 2008; 40(4): 112-129.

9. Arnetz BB. Psychosocial challenges facing physicians of today. Soc Sci Med. 2001; 42(2): 203213. doi: 10.1016/S0277-9536(00)00220-3

10. Rogers AE, Hwang WT, Scott LD, et al. The working hours of hospital staff nurses and patient safety. Health Aff. 2004; 23(4): 202-212. doi: 10.1377/hlthaff.23.4.202

11. Juárez-garcía A. Factores psicosociales, estrés y salud en distintas ocupaciones: un estudio exploratorio. Investig en Salud 2007; 9: 57-64.

12. Organización Internacional del Trabajo. Frente a la pandemia: garantizar la seguridad y salud en el trabajo, https://www.ilo.org/wcmsp5/groups/ public/---ed_protect/---protrav/---safework/ documents/publication/wcms_742732.pdf

13. Wu PE, Styra R, Gold WL. Mitigating the psychological effects of COVID-19 on health care workers. CMAJ. 2020; doi: 10.1503/cmaj.200519

14. Tsamakis K, Rizos E, Manolis A, et al. COVID-19 pandemic and its impact on mental health of healthcare professionals. Exp Ther Med. 2020. doi: 10.3892/etm.2020.8646
15. Lai J, Ma S, Wang Y, Cai Z, Hu J, Wei N, et al. Factors associated with mental health outcomes among health care workers exposed to coronavirus disease 2019. JAMA Netw open. 2020; 3(3): e203976. doi: 10.1001/jamanetworkopen.2020.3976

16. El-Hage W, Hingray C, Lemogne C, Yrondi A, Brunault P, Bienvenu T, et al. Health professionals facing the coronavirus disease 2019 (COVID-19) pandemic: What are the mental health risks? Encephale. 2020; 46: S73-S80.

17. Huang Y, Zhao N. Generalized anxiety disorder, depressive symptoms and sleep quality during COVID-19 outbreak in China: a web-based crosssectional survey. Psychiatry Res. 2020; doi: 10.1016/j.psychres.2020.112954

18. Rossi R, Socci V, Pacitti F, Di Lorezo G, Di Marco A, Siracusano A, et al. Mental health outcomes among front and second line health workers associated with the COVID-19 pandemic in Italy. medRxiv. 2020; doi: 10.1101/2020.04.16.20067801

19. Tan BYQ, Chew NWS, Lee GKH, Jing M, Goh Y, Yeo L, et al. Psychological Impact of the COVID-19 Pandemic on health care workers in Singapore. Ann Intern Med. 2020; doi: 10.7326/m20-1083

20. Chen Q, Liang M, Li Y, et al. Mental health care for medical staff in China during the COVID-19 outbreak. Lancet Psychiatry. 2020; doi: 10.1016/ S2215-0366(20)30078-X

21. Toribio L. Coronavirus: 'Personal de salud se siente en riesgo': encuesta de la UNAM. Excelsior, https://www.excelsior.com.mx/nacional/personalde-salud-se-siente-en-riesgo-encuesta-de-launam $/ 1387526$

22. Morelos C de DH del E de. Encuesta para el personal de salud de Morelos. Cuernavaca, 2018.

23. Juárez-García A. Burnout en profesionales de la salud durante la pandemia. En: Ciclo de videoconferencias: Estrategias de acción para el bienestar psicosocial en tiempos de COVID-19. Centro de Investigación Transidsicplinar en PsicologíaUAEM. 2020.

24. World Health Organization. WHO | Burn-out an "occupational phenomenon": International Classification of Diseases, https://www.who.int/ mental_health/evidence/burn-out/en/

25. Maslach C. Burnout: A multidimensional perspective. En: Professional burnout: Recent developments in theory and research. Philadelphia, PA, US: Taylor \& Francis, 1993, pp. 19-32.

26. Büssing A, Glaser J. Four-stage process model of the core factors of burnout: The role of work stressors and work-related resources. Work Stress 2000; 14: 329-346. 
27. Leiter MP, Maslach C. Latent burnout profiles: A new approach to understanding the burnout experience. Burn Res 2016; 3: 89-100.

28. Russell JA. A circumplex model of affect. J Pers Soc Psychol. Epub ahead of print 1980. doi: 10.1037/ h0077714

29. Bakker AB, Oerlemans WGM. Subjective Wellbeing in Organizations. En: The Oxford Handbook of Positive Organizational Scholarship. 2012; doi: 10.1093/oxfordhb/9780199734610.013.0014

30. World Health Organization (WHO). International Classification of Diseases (ICD-11) - Mortality and Morbidity Statistics. World Health Organization, https://icd.who.int/browse11/1-m/en\#/http://id.who. int/icd/entity/129180281

31. Juarez Garcia A. Entrevista Con Christina Maslach: Reflexiones sobre el síndrome de burnout. Lib Rev Psicol.

32. García AJ, Gil-Monte PR, Merino-Soto C, et al. From a polemic paradox to a proper perspective of job burnout and job satisfaction. Journal of Neurosurgery. 2019; doi: 10.3171/2018.10. JNS183014

33. Arias Galicia F, Juárez García A. Agotamiento profesional y estrés: hallazgos desde México y otros países latinoamericanos. México: Miguel Ángel Porrúa.

34. Salvagioni DAJ, Melanda FN, Mesas AE, et al. Physical, psychological and occupational consequences of job burnout: A systematic review of prospective studies. PLoS ONE. 2017; doi: 10.1371/journal.pone.0185781

35. Tsiga E, Panagopoulou E, Montgomery A. Examining the link between burnout and medical error: A checklist approach. Burn Res. Epub ahead of print 2017. doi: 10.1016/j.burn.2017.02.002

36. Halbesleben JRB, Rathert C. Linking physician burnout and patient outcomes. Health Care Manage Rev 2008; 33: 29-39.

37. Selye H. A syndrome produced by diverse nocuous agents. Nature. Epub ahead of print 1936. doi: 10.1038/138032a0

38. Vignolo Ballesteros J, Vacarezza Consani M, Álvarez Yunti C, et al. Niveles de atención, de prevención y atención primaria de la salud. Arch med interna.

39. Babaian J. The Pandemic's 4th Wave | hcldr. Web Page, https://hcldr.wordpress.com/2020/04/07/thepandemics-4th-wave/

40. Kancherla BS, Upender R, Collen JF, et al. Sleep, fatigue and burnout among physicians: an American Academy of Sleep Medicine position statement. J Clin Sleep Med 2020; 16: 803-805.
41. Sasangohar F, Jones SL, Masud FN, et al. Provider Burnout and Fatigue During the COVID-19 Pandemic: Lessons Learned From a High-Volume Intensive Care Unit. Anesth Analg 2020; 131: 106-111.

42. Sultana A, Sharma R, Hossain MM, et al. Burnout among healthcare providers during COVID-19: Challenges and evidence-based interventions. Indian J Med Ethics 2020; 01-04.

43. Bradley M, Chahar P. Burnout of healthcare providers during COVID-19. Cleve Clin J Med. Epub ahead of print el 9 de julio de 2020. doi: 10.3949/ccjm.87a.ccc051

44. Leiter MP, Maslach C. Areas of worklife: a structured approach to organizational predictors of job burnout. Res Occup Stress Well Being. 2003; 3: 91-134.

45. LaMontagne AD, Keegel T, Louie AM, et al. A systematic review of the job-stress intervention evaluation literature, 1990-2005. International J Occup Environm Health 2007; 13: 268-280.

46. Fessell D, Cherniss C. Coronavirus Disease 2019 (COVID-19) and Beyond: Micropractices for Burnout Prevention and Emotional Wellness. J Am College Radiol. 2020; doi: 10.1016/j. jacr.2020.03.013.

47. Lozano-Vargas A. Impacto de la epidemia del Coronavirus (COVID-19) en la salud mental del personal de salud y en la población general de China. Rev Neuropsiquiatr. 2020; doi: 10.20453/ rnp.v83i1.3687

48. Ornell F, Halpern SC, Paim Kessler FH, et al. The impact of the COVID-19 pandemic on the mental health of healthcare professionals. Cad Saude Publica. 2020; 36: 63520.

49. The Lancet. COVID-19: protecting health-care workers. The Lancet 2020; 395: 922. 